\title{
Probing the kinetics in Supramolecular Chemistry and Molecular Assembly by Microfluidic-NMR Spectroscopy
}

\author{
Hongxun Fang ${ }^{1}$, Yibin Sun ${ }^{1}$, Xinchang Wang ${ }^{12}$, Manvendra Sharma ${ }^{3}$, Xiaoyu Cao ${ }^{1 *}$, Marcel Utz ${ }^{3 *}$, \\ Zhongqun Tian"
}

\author{
'State Key Laboratory of Physical Chemistry of Solid Surfaces, College of Chemistry and Chemical Engineering, Collaborative Innovation \\ Center of Chemistry for Energy Materials and Key laboratory of Chemical Biology of Fujian Province, Xiamen University, Xiamen 361005, \\ China. \\ DDepartment of Electronic Science, Fujian Provincial Key Laboratory of Plasma and Magnetic Resonance, Xiamen University, Xiamen 361005, \\ China \\ School of Chemistry, University of Southampton, SO17 1BJ, UK.
}

Received April 12, 2018; accepted xxxx, 2018; published online xxxx, 2018

\begin{abstract}
Microfluidic-NMR spectroscopy has been extended to study the kinetics in supramolecular chemistry and molecular assembly. Kinetics of a multicomponent host-guest supramolecular system containing viologen derivatives, $\beta$-cyclodextrins and Cucrurbit[7]urils are studied by a PMMA based microfluidic chip combined with a dedicated transmission line probe for NMR detection. By combining microfluidic technology with NMR spectroscopy, the amount of material required for a full kinetic study could be minimized. This is crucial in supramolecular chemistry, which often involves highly sophisticated and synthetically costly building blocks. The small size of the microfluidic structure is crucial in bringing the time scale for kinetic monitoring down to seconds. At the same time, the transmission line NMR probe provides sufficient sensitivity to work at low (2 mM) concentrations.
\end{abstract}

microfluidic-NMR, molecular assembly, host-guest chemistry, kinetics

\section{Introduction}

Molecular assembly provides a bottom-up method to construct sophisticated supramolecular structures and complex functional materials[1-2]. The scope of molecular assembly expands from small molecular to biological macromolecular systems, involving hierarchical[3], multicomponent assembly processes[4] and pathway complexity[5]. The rational design and construction of functional supramolecular systems that mimic biology[6] require a thorough under- standing of the structures of assemblies at thermodynamic equilibrated state as well as the kinetics of the assembly processes. The structures can now be characterized at high-resolution by X-ray crystallography[7], nuclear magnetic resonance (NMR) spectroscopy[8-9], mass spectroscopy (MS)[10] etc. The current techniques to characterize the kinetic processes of molecular assembly[11-13], however, remain insufficient to provide information with high-resolution in real time.

*Corresponding authors (email: zqtian@xmu.edu.cn, Marcel.Utz@soton.ac.uk,xcao@xmu.edu.cn ) 
NMR spectroscopy is a powerful technique that provides detailed structural information on the supramolecular systems at both the molecular and supramolecular level[9, 14], which allows quantitative analysis and non-invasive in-situ monitoring. Although NMR spectroscopy can characterize the kinetics of relatively slow molecular assembly processes (on timescales of hours to days), poor sensitivity often requires long acquisition times for a single NMR spectrum, thus precluding the monitoring of kinetic processes on timescales of minutes or less. Miniaturized NMR coils offer significant improvements in mass sensitivity [1]. Flow probes based on solenoid microcoils are commonly used in hyphenated systems that combine NMR with separation techniques such as HPLC or capillary electrophoresis. [2,3] Miniaturized continuous-flow NMR systems can improve both sensitivity and time resolution, as demonstrated by Ciobanu et al.[16], who wrapped a capillary with small solenoidal coil and used it as miniaturized continuous-flow NMR. Since then, more sensitive miniaturized NMR detectors [17] and flow techniques [18] have been introduced to NMR spectroscopy and used to monitor the kinetics of chemical reactions.

However, the kinetic study of molecular assembly imposes two essential requirements on the NMR spectroscopy: the concentration and the volume of sample during the measurement. First, the concentrations of molecular assemblies are generally low (in most cases $10 \mathrm{mM}$ or lower). Second, sample consumption during the kinetic measurement should be minimized because the building blocks of molecular assembly are often highly sophisticated (and thus expensive). Due to the inherently low sensitivity of NMR spectroscopy, both requirements are hard to fulfil at the same time. The reported work involving kinetic studies through NMR techniques had to compromise one or two of these requirements. On one hand, the consumption of sample ( $\mathrm{pL}$ to $\mu \mathrm{L}$ ) required by miniaturized continuous-flow NMR technique is small, but the concentrations of samples need to be high (above $100 \mathrm{mM}$ )[18-20]. On the other hand, increasing the volume of samples allowed the concentration to be lowered to $1 \mathrm{mM}$ using a stopped-slow NMR technique[21], but the great demand for samples (typically $600 \mu 1$ per measurement) prevents its further utilization in molecular assembly. In general, monitoring the kinetic processes of molecular assembly through NMR spectroscopy with little sample consumption at low concentration is still challenging.

In this contribution, we monitor the kinetics of a molecular process using a microfluidic chip reactor integrated with an optimized double-stripline NMR probe head [22]. The double-stripline detector focuses the radio-frequency (RF) magnetic field onto a NMR sample chamber on the microfluidic chip with a total volume of $5 \mu \mathrm{L}$. The molecular assembly process involves dethreading of curcubit[7]uril (CB7) from viologen derivatives by adding an adamantanamine hydrochloride (Ad) derivative. The two components for the molecular assembly process are intro- duced into the microfluidic chip using syringe pumps and

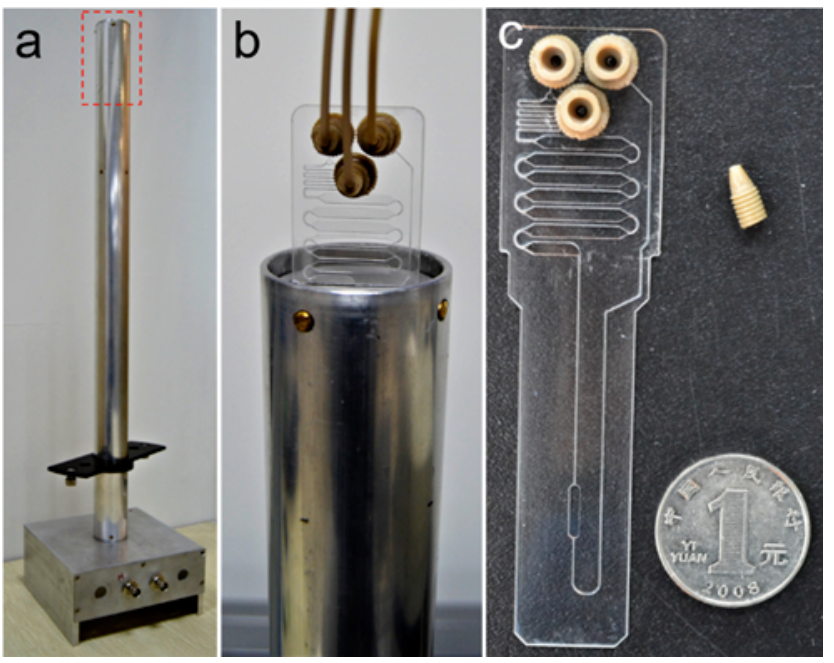

mixed inside the chip. By tuning the flow rates, in-situ NMR spectra are taken at different residence times (from 15 seconds to 10 minutes). Our work demonstrates the first NMR investigation of the kinetic process of molecular assembly at low concentration $(2 \mathrm{mM})$ with minimum sample consumption.

Figure. 1 (a) The microfluidic NMR probe inside NMR probe. The dashed line indicates the position of the chip. (b) Close-up view of the chip inserted in the probe. (c) Close-up view of the chip with coned nano port.

\section{Experimental}

\subsection{Nuclear magnetic resonance device set up}

The microfluidic-NMR experiments in this work were carried out using a Varian NMR system operating at a proton Larmor frequency of $500 \mathrm{MHz}$. The original probe was replaced with our custom-built transmission line NMR probe head (Figure. 1a). The radio-frequency magnetic field transmitted by the double-stripline is concentrated at the rectangular sample chamber to ensure enough sensitivity. More detailed information about the NMR probe head can be found in Ref. [22].

\subsection{Fabrication of microfluidic chips}

The microfluidic chip was fabricated following a procedure described elsewhere[23]. Poly(methyl methacrylate) (PMMA) sheets (Clarex Precision sheets, $0.5 \mathrm{~mm}$ and 0.2 $\mathrm{mm}$ ) were purchased from Nitto Jushi Kogyo Co., Ltd. The microfluidic chip consists of three PMMA layers: the middle layer $(0.5 \mathrm{~mm})$ was cut through using a laser engraver to give channels and chambers; the other two layers $(0.2 \mathrm{~mm}$ each) were used to seal the chip. The three layers were bonded together to give a total thickness of $0.9 \mathrm{~mm}$. The chip was $99.1 \mathrm{~mm}$ long and $25.8 \mathrm{~mm}$ wide. The assembled chip can be inserted into the NMR probe (Figure. 1b) and is 
exchangeable for different assembly systems.

\subsection{Pumping of reactants}

The reactants were pumped into chip through tubes driven by syringe pumps outside the NMR magnet. The tubes that connecting syringe pumps and chip were made of polyether ether ketone (PEEK) with an outer diameter (OD) of 1/16 inch. The inner diameter (ID) of tubes connecting inlet and outlet channels are different. To reduce dead volume from pump to chip, we use PEEK tubes with ID of 0.007 inch to connect the pump and inlet channels; to reduce the back pressure of chip, we use PEEK tube with ID of 0.04 inch to connect outlet channel. The tubes and chip were connected through commercially available PEEK coned nano ports (Figure. 1c). The total volume of the fluids inside the fluidic system was experimentally measured to be around $350 \mu \mathrm{L}$.

\subsection{Mixing efficiency of fluids in microfluidic chip}

The reactants were mixed inside the chip at the Y-junction inside the chip (Figure. 2). To illustrate the mixing efficiency of chip, we mixed two fluids in different colors: the transparent fluid is pure water, and the pink fluid is aqueous solution of potassium permanganate $(5 \mathrm{mM})$. As Figure. S3 shows, the two fluids were mixed efficiently at the end of mixing channel even at the highest flow rate $(150 \mu \mathrm{L} / \mathrm{min}$ for each channel) in this work.

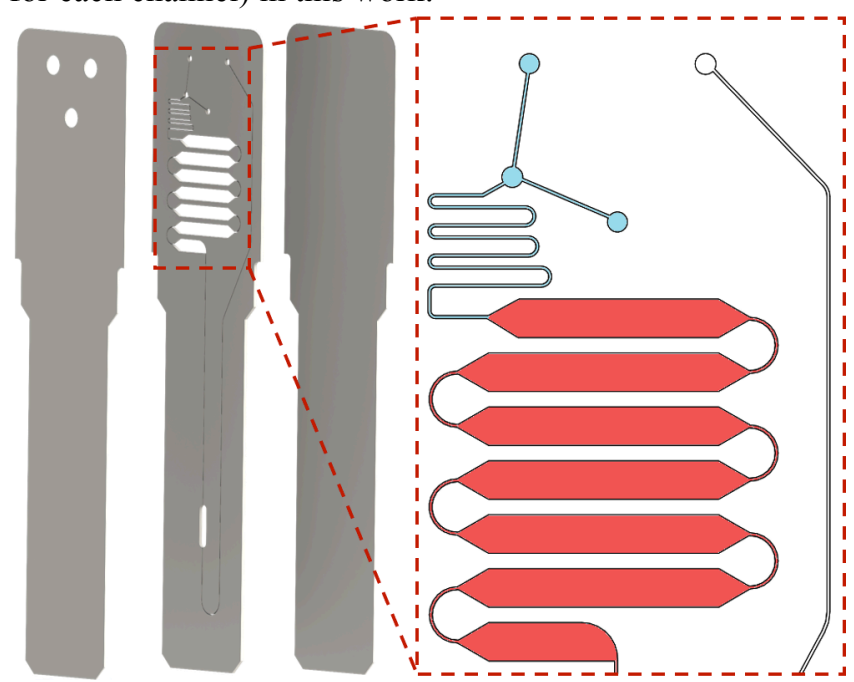

Figure. 2 Exploded view of the microfluidic chip (left). The chip is composed of three layers of PMMA. Solution of complex A and B were injected through coned nano port into the chip and mixed in mixing channel (in blue dashed box) in the middle layer. Then the mixture transported through the wide channel (in red dashed box) and eventually got the sample chamber.

\subsection{Buffering chambers and flow rate}

To monitor the kinetic process by continuos-flow, the residence time at the NMR detecting area should be tuned to the time scale of the kinetic process. For a microfluidic chip, the residence time is determined by two parameters: the volume of the chamber before the detecting area and the flow rate. Because the flow rate can be easily tuned through syringe pump, we fixed the volume of chambers before the NMR detecting area to $75 \mu \mathrm{L}$, located between the end of mixing channel and the detecting area. Hence, by changing the flow rate, the residence time at the NMR detecting area can be precisely controlled.

Moreover, because the NMR detection chamber had a total volume of $5 \mu \mathrm{L}$, the time for fluid to flow through it should also be taken into consideration. To achieve high time resolution, the flow rate should be tuned within a reasonable range. If the flow rate is too low, the difference of residence time between the front and back part of the sample chamber is long, leading to poor time resolution. However, if the flow rate is too high, the sample would be flushed out of the NMR detection area before signal acquisition is complete, broadening and diminishing the NMR spectra. As a result, the flow rate for each syringe pump was set from 7.5 $\mu \mathrm{L} / \mathrm{min}$ to $150 \mu \mathrm{L} / \mathrm{min}$, corresponding to residence times in the reaction chamber from 5 minutes down to 15 seconds.

\subsection{Molecular assembly system}

The viologen derivative (didodecyl-4,4'-bipyridinium dibromide) was prepared following the literature[24]. All reagents were purchased from Sigma-Aldrich and J\&K and used without further purification. Aqueous solutions were prepared using $\mathrm{D}_{2} \mathrm{O}$.

Host-guest chemistry is an efficient approach to construct complex supramolecular structures. In this work, we applied our microfluidic NMR technique to monitor the kinetic process of a host-guest process based on cucurbit[7]urils $(\mathrm{CB}[7]), \beta$-cyclodextrins ( $\beta$-CDs) and viologen derivatives (Figure. $3 \mathrm{a}$ ). The assembly process involves mixing of two supramolecular complexes A and B. Complex A (4 mM in $\left.\mathrm{D}_{2} \mathrm{O}\right)$ is a rotaxane containing a viologen derivative with $n$-dodecyl chain (guest) threading four $\beta$-CDs[25] and one $\mathrm{CB}$ [7] (host)[26]. The structure of $\mathbf{A}$ has been validated by 2D-ROESY (Figure. S1) and isothermal titration calorimetry (ITC) (Figure S2). Complex B (4 mM in $\left.\mathrm{D}_{2} \mathrm{O}\right)$ is a host-guest complex of $\beta$-CDs (host) and adamantanamine hydrochloride (Ad) (guest) in 1:1 ratio. Upon mixing of the two complexes through two inlet channels, CB[7] dethreaded out of complex A and extracted Ad out of complex $\mathbf{B}$, because the binding constant between $\mathrm{CB}[7]$ and $\mathrm{Ad}$ is eight orders of magnitude larger than that between $\beta-C D$ and $\operatorname{Ad}[27-28]$. After completion of this assembly process, $\mathbf{A}$ turned into $\mathbf{C}$ and $\mathbf{B}$ turned into D.This assembly process involves a series of disassembly and re-assembly steps, which take about ten minutes to finish at $2 \mathrm{mM}$.

\section{Results and discussion}

The two complexes were mixed inside the chip and in-situ NMR spectra were taken at the NMR detecting area. Each ${ }^{1} \mathrm{H}-\mathrm{NMR}$ spectrum was acquired by accumulating 128 scans within 10 minutes. A perfect spin echo is used to remove the 
signal of solid materials in microfluidic chip (mainly PMMA)[29]. The quality of each spectrum varied with flow rates: the signal-to-noise (SNR) ratio varied from 1000 to 2700, and the line width varied with full-width-at-half-maximum (FWHM) ranging from 0.0253 ppm $(12.645 \mathrm{~Hz})$ to $0.0128 \mathrm{ppm}(6.391 \mathrm{~Hz})$. In addition, comparison between spectrum taken from commercial probe and spectrum taken from our microfluidic probe was
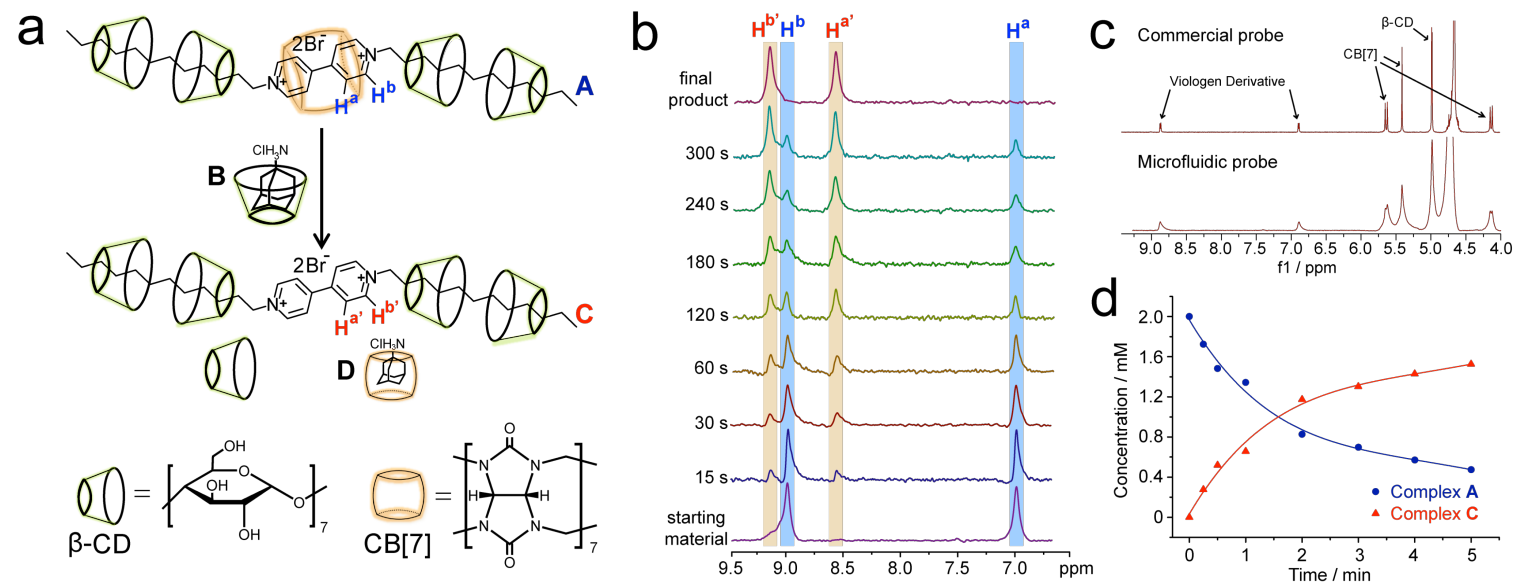

Figure. 3 (a) Host-guest process based on viologen derivative and Ad. (b) Observed 1H NMR spectra as a function of residence time. (c) ${ }^{1} \mathrm{H}$ NMR spectra of $2 \mathrm{mM}$ complex A taken from commercial probe and microfluidic probe. (d) Kinetic curve extracted from ratio of integrated peak area between complex A and complex $\mathbf{C}$

made. As is shown in Figure. 3c, viologen derivative, CB[7] and $\beta$-CD could be identified easily.

The kinetic curves of this assembly process were drawn by integrating two aromatic protons from viologen derivative during the assembly process. $\mathrm{CB}[7]$, being an aromatic macrocycle, induces diamagnetic shielding to its guest molecule. Therefore, after $\mathrm{CB}[7]$ was dethreaded from $\mathbf{A}$, the aromatic protons in the viologen derivative appear shifted to higher fields. Hence, by integrating the aromatic protons of $\mathrm{H}^{\mathrm{a}}$ and $\mathrm{H}^{\mathrm{b}}$ (Figure. 3b), the relative concentration of complex $\mathbf{A}$ and $\mathbf{C}$ can be determined. As shown in Figure. $3 \mathrm{~d}$, the kinetic curve is plotted as the ratio of each set of peak area to the sum of both sets, as a function of residence time.

However, this curve cannot be fitted using first order nor second order reaction mechanism. We suspect that this assembly process involves several disassembly and re-assembly steps, and may thus require more complex kinetic models. Another possible source that may contribute to this finding is the uneven flow velocity profile associated with laminar flow. Fluid moving through the centre of the channels does so at higher velocity than close to the channel walls. Such effects can be captured by finite element simulations of the combined flow/reaction/diffusion processes in the device. Such calculations are underway in our laboratory, and will be reported on later.

\section{Conclusions}

In summary, this work demonstrated the possibility of using NMR as an analytical tool to study molecular assembly kinetics of limited sample concentration with reaction times of a few minutes by using microfluidic
NMR with the optimized double-stripline microcoil and corresponding PMMA chip. Continuous flow decoupled the total spectra-measurement time from the reaction time, and the enlarged detecting area and sample chamber significantly improved the concentration limit of detection. Monitoring of the molecular assembly kinetics of host-guest interaction between viologen derivative, $\mathrm{CB}[7]$ and $\beta$-CDs at $2 \mathrm{mM}$ concentration was demonstrated, and was compared with measurements in a conventional tube in a $500 \mathrm{MHz}$ NMR system. The kinetic curve didn't fit first order nor second order reaction mechanism, indicating this host-guest interaction involves multiple equilibriums. The reaction time in the chip can be set down to seconds, thus enabling monitoring of relatively fast kinetics. A further expansion of the double-stripline probe head to include another frequency channel $\left({ }^{13} \mathrm{C}\right.$ or $\left.{ }^{15} \mathrm{~N}\right)$ is possible[30], which allows 2D heteronuclear and multi-nuclear NMR experiments and have a great potential in structure determination of unstable reaction intermediates, protein folding kinetics studies and interaction studies between biomolecules and small molecules.

Acknowledgments We thank Billy Hale for his help on the bonding protocol of microfluidic chip. We thank Chen Zhong for providing the accessibility to superconducting magnet. We thank Chen Jia-He for his help on NMR data acquirement. This work was supported by the 973 Program (No. 2015CB856500), the NSFC (Nos. 21722304, 21573181, 91227111, and 91427304) and the Fundamental Research Funds for the Central Universities of China (No. 20720160050). Development and construction of the NMR probe was supported in part by a Marie Curie Career Integration Grant to MU by the European Commission (Project uf-NMR).

Conflict of interest The authors declare that they have no conflict of interest. 
Supporting information The supporting information is available online at http://chem.scichina.com and http://link.springer.com/journal/xxxx. The supporting materials are published as submitted, without typesetting or editing. The responsibility for scientific accuracy and content remains entirely with the authors.

1. Zhang X, Zeng Q, Wang C. Sci. China. Chem., 2014, 57: 13-25.

2. Lehn JM. Angew. Chem. Int. Ed., 2013, 52: 2836-2850.

3. Elemans JA, Rowan AE, Nolte RJ. J. Mater. Chem., 2003, 13: 2661-2670.

4. Ludlow RF, Otto S. Chem. Soc. Rev., 2008, 37: 101-108.

5. Korevaar PA, George SJ, Markvoort AJ, Smulders MM, Hilbers PA, Schenning AP, De Greef TF, Meijer E. Nature, 2012, 481: 492.

6. Bohne C. Chem. Soc. Rev., 2014, 43: 4037-4050.

7. Enright GD, Udachin KA, Ripmeester JA. CrystEngComm, 2010, 12: 1450-1453.

8. Clore GM, Gronenborn AM. Proc Natl Acad Sci, 1998, 95: 5891-5898.

9. Schneider H-J, Hacket F, Rüdiger V, Ikeda H. Chem. Rev., 1998, 98: 1755-1786.

10. Jiang W, Nowosinski K, Löw NL, Dzyuba EV, Klautzsch F, Schäfer A, Huuskonen J, Rissanen K, Schalley CA. J. Am. Chem. Soc., 2012, 134: 1860-1868.

11. Wojciechowski JP, Martin AD, Thordarson P. J. Am. Chem. Soc., 2018.

12. Yang J, Wu K, Koňák Ce, Kopecek J. Biomacromolecules, 2008, 9: 510-517.

13. Cai W, Wang G-T, Du P, Wang R-X, Jiang X-K, Li Z-T. J. Am. Chem. Soc., 2008, 130: 13450-13459.

14. Grzesiek S, Sass H-J. Curr Opin Struc Biol, 2009, 19: 585-595.

15. Abragam A, The principles of nuclear magnetism. Oxford university press: 1961.

16. Ciobanu L, Jayawickrama DA, Zhang X, Webb AG, Sweedler JV. Angew. Chem., Int. Ed., 2003, 42: 4669-4672, S4669/1-S4669/2.

17. Badilita V, Meier RC, Spengler N, Wallrabe U, Utz M, Korvink JG. Soft Matter, 2012, 8: 10583-10597.

18. Bart J, Kolkman AJ, Oosthoek-de Vries AJ, Koch K, Nieuwland PJ, Janssen H, van Bentum J, Ampt KA, Rutjes FP, Wijmenga SS. J. Am. Chem. Soc., 2009, 131: 5014-5015.

19. Wensink H, Benito-Lopez F, Hermes DC, Verboom W, Gardeniers HJ, Reinhoudt DN, van den Berg A. Lab. Chip., 2005, 5: 280-284.

20. Gomez MV, Rodriguez AM, de la Hoz A, Jimenez-Marquez F, Fratila RM, Barneveld PA, Velders AH. Anal. Chem., 2015, 87: 10547-10555.

21. Christianson MD, Tan EHP, Landis CR. J. Am. Chem. Soc., 2010, 132: 11461-11463.

22. Finch G, Yilmaz A, Utz M. J. Magn. Reson, 2016, 262: 73-80.

23. Yilmaz A, Utz M. Lab. Chip., 2016, 16: 2079-2085.

24. Nieto-Ortega B, Villalva J, Vera-Hidalgo M, Ruíz-González L, Burzurí E, Pérez EM. Angew. Chem. Int. Ed., 2017.

25. Jiang L, Peng Y, Yan Y, Huang J. Soft Matter, 2011, 7: 1726-1731.

26. Han Y, Gao C, He X. Sci. China. Chem., 2012, 55: 604-611.

27. Rekharsky MV, Inoue Y. Chem. Rev., 1998, 98: 1875-1917.

28. Barrow SJ, Kasera S, Rowland MJ, del Barrio J, Scherman OA. Chem. Rev., 2015, 115: 12320-12406.

29. Takegoshi K, Ogura K, Hikichi K. Journal of Magnetic Resonance (1969), 1989, 84: 611-615.

30. Bart J, Janssen J, Van Bentum P, Kentgens A, Gardeniers JG. J. Magn. Reson, 2009, 201: 175-185.
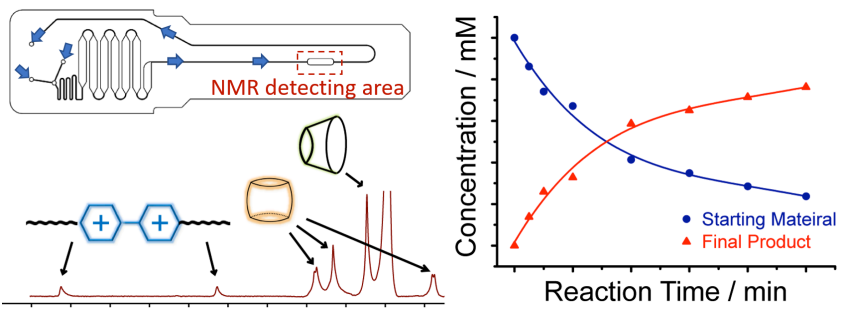

[1] N. Wu, T.L. Peck, A.G. Webb, R.L. Magin, J.V. Sweedler, Nanoliter Volume Sample cells for $1 \mathrm{H}$ NMR: Application to Online Detection in Capillary Electrophoresis, J. Am. Chem. Soc. 116 (1994) 7929-7930. doi:10.1021/ja00096a073.

[2] R. Subramanian, W. Kelley, P. Floyd, Z. Tan, A. Webb, J. Sweedler, A Microcoil NMR Probe for Coupling Microscale HPLC with On-Line NMR Spectroscopy, Anal. Chem. 68 (1996) 4431-4435.

[3] Klaus Pusecker, Jens Schewitz, Petra Gfrörer, Li-Hong Tseng, A. Klaus Albert, E. Bayer, On-Line Coupling of Capillary Electrochromatography, Capillary Electrophoresis, and Capillary HPLC with Nuclear Magnetic Resonance Spectroscopy, Analytical .... 70 (1998) 3280-3285. doi:10.1021/ac980063o.

\section{Table of Contents graphic}

